\title{
Robust Silk Fibroin/Graphene Oxide Aerogel Fiber for Radiative Heating Textiles
}

Zongqian Wang*, Haiwei Yang, Yu Li and Xianhong Zheng*

School of Textile and Garment, Anhui Polytechnic University, Wuhu, 241000, China;

\section{Corresponding author:}

Dr. Xianhong Zheng (E-mail: zhengxianhong@ahpu.edu.cn)

Prof. Zongqian Wang (E-mail: wzqkeyan@126.com) 

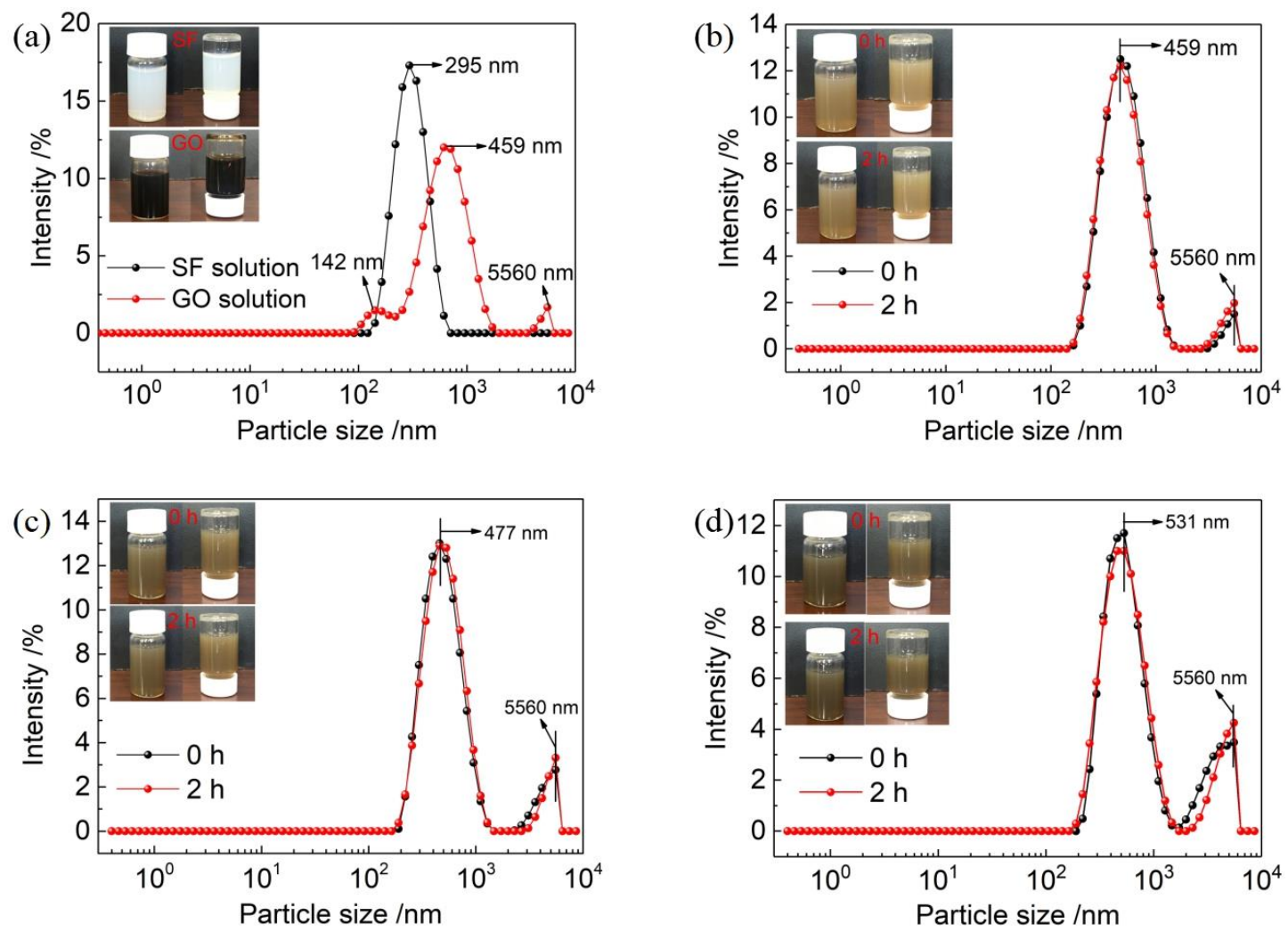

Figure S1. (a) Particle size distribution of SF and GO solutions; particle size distribution of (b) SF / GO 1.0, (c) SF / GO 1.5, and (d) SF / GO 2.0 solution. 

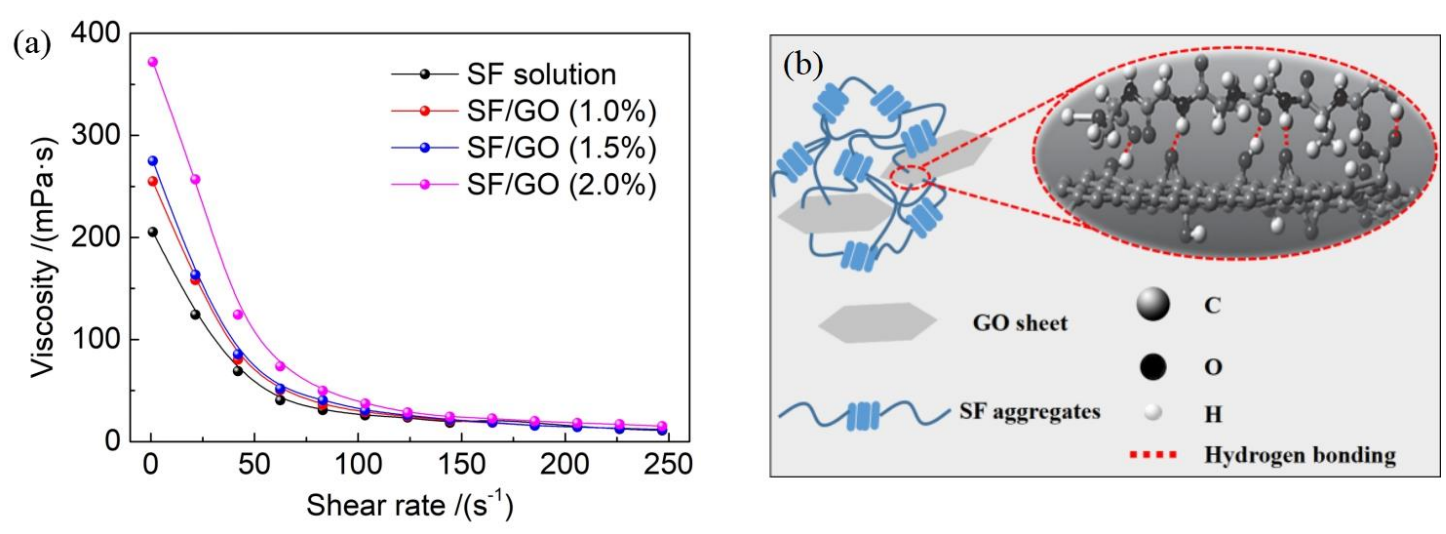

Figure S2. (a) Rheology curves of SF and SF/GO solution, (b) schematic illustration of interactions between SF and GO. 

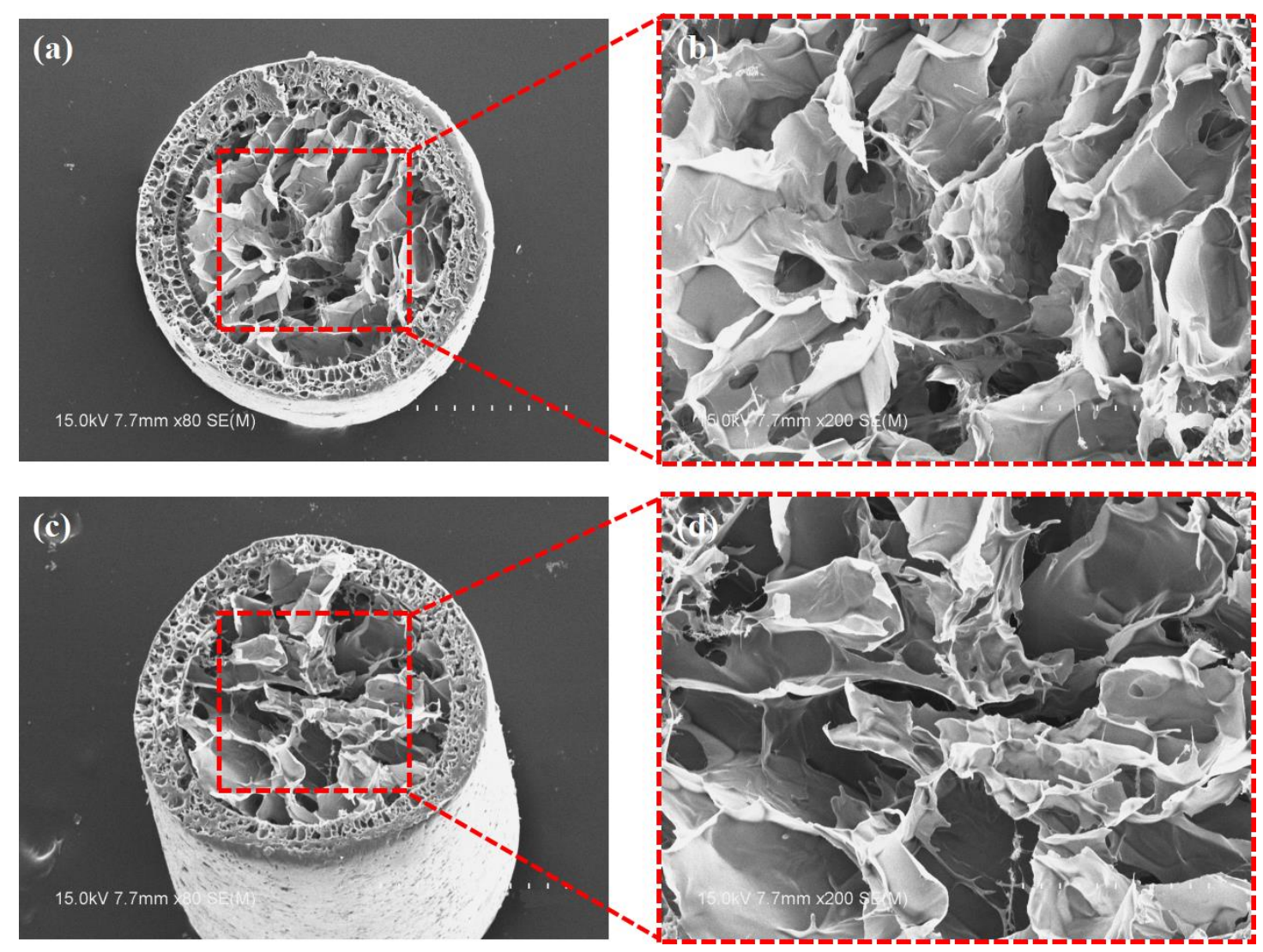

Figure S3. ( $a$ and b) SEM images of SF/GO 1.0 aerogel fiber cross section, (C and D) SEM images of SF/GO 1.5 aerogel fiber cross section 


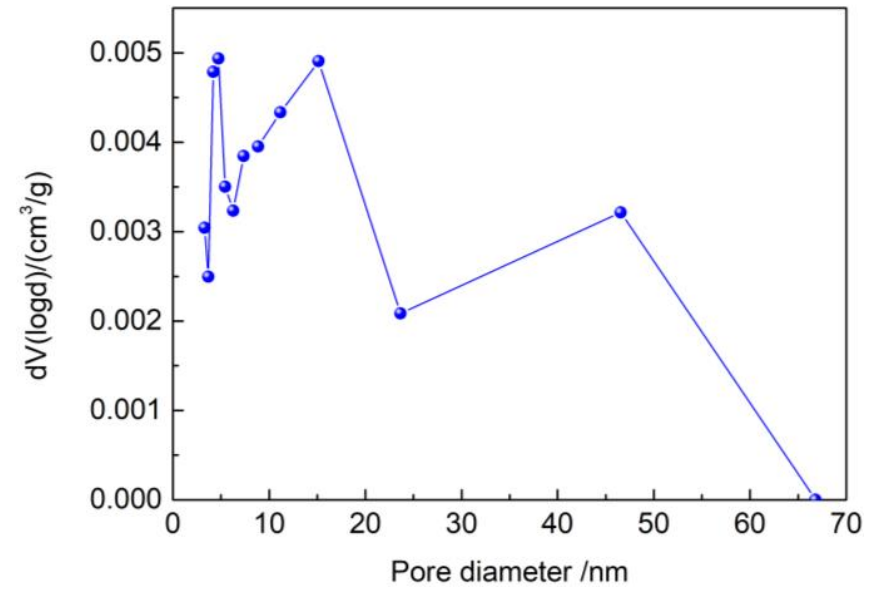

Figure S4. Pore size distribution of SF aerogel measured by Barrett-Joyner-Halenda method 
The density of the SF and SF/GO 2.0 aerogel core were calculated from the mass difference between the aerogel fiber and the hollow fiber of equal lengths and the diameter of the SF and SF/GO 2.0 aerogel core measured from SEM images to be 12.7 and $13.6 \mathrm{mg} / \mathrm{cm}^{3}$, respectively. Meanwhile, the porosity of SF and SF/GO 2.0 aerogel core was calculated according to equation (S1) and equation (S2), respectively, as shown in Figure S4.

$$
P_{1}(\%)=\left(1-\frac{\rho_{1}}{\rho_{S F}}\right) \times 100 \% \quad \text { (Equation S1) }
$$

where $P_{1}$ is porosity of the SF aerogel core, $\rho_{1}$ is density of the SF aerogel core and $\rho_{\mathrm{SF}}$ is the bulk density of SF equals $1.464 \mathrm{~g} / \mathrm{cm}^{3} .{ }^{1}$

$$
P_{2}(\%)=\left[1-\left(\frac{\rho_{2} \times \frac{x}{51}}{\rho_{\mathrm{SF}}}\right)-\left(\frac{\rho_{2} \times \frac{y}{51}}{\rho_{\text {Graphite }}}\right)\right] \times 100 \% \quad \text { (Equation S2) }
$$

where where $P_{2}$ is porosity of the $\mathrm{SF} / \mathrm{GO} 2.0$ aerogel core, $\rho_{2}$ is density of the SF/GO 2.0 aerogel core and $\rho_{\text {Graphite }}$ is the bulk density of Graphite equals 2.25 $\mathrm{g} / \mathrm{cm}^{3}, \frac{\mathrm{x}}{51}$ is the weight ratio of SF in an aerogel, and $\frac{\mathrm{y}}{51}$ is the weight ratio of GO in an aerogel. Among them, $x / y=50 / 1$.

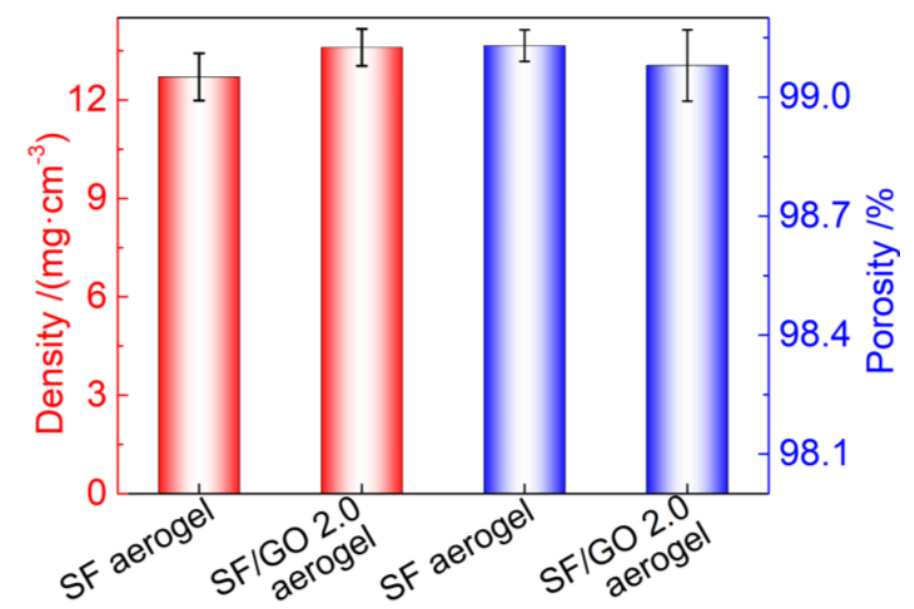

Figure S5 Density and porosity of SF and SF / GO 2.0 aerogel cores in hollow fibers 


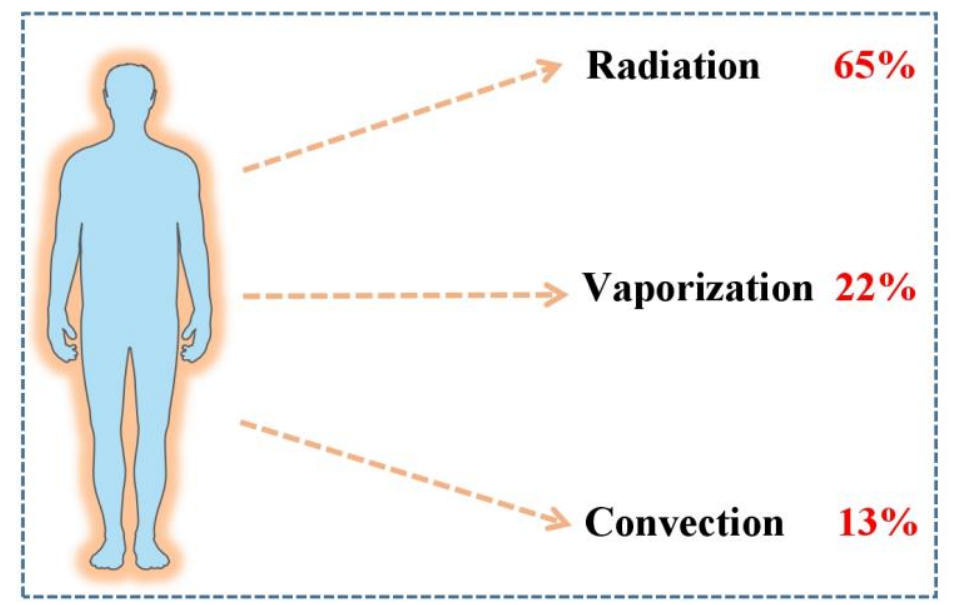

Figure S6. Schematic of human body heat loss distribution at ambient temperature of $26{ }^{\circ}{ }^{3}{ }^{3}$ 


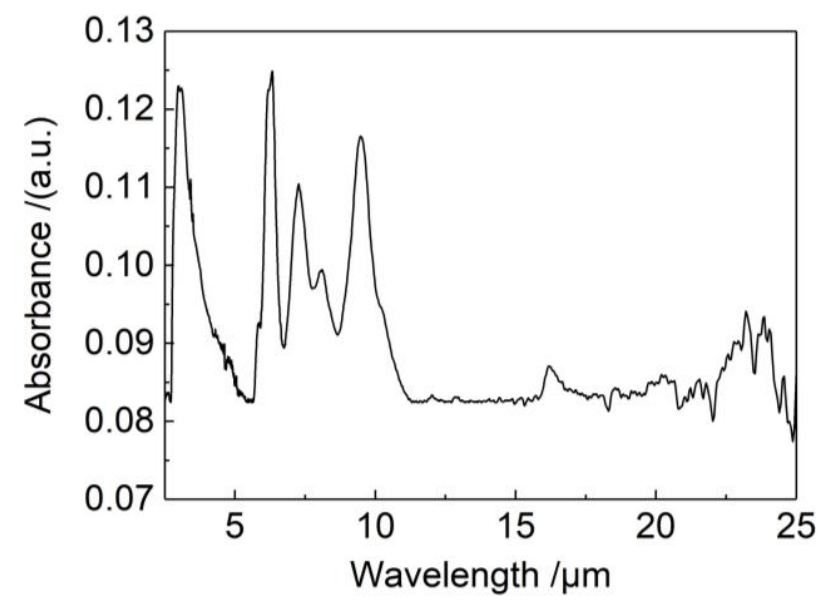

Figure S7. FTIR absorbance spectra of GO aerogel

\section{References}

(1) Frey-Wyssling, A. J. B. e. b. a. On the density and the optics of silk fibroin. Bba-Biomembranes 1955, 17 (1), 155-156.

(2) Rattanaweeranon, S.; Limsuwan, P.; Thongpool, V.; Piriyawong, V.; Asanithi, P. J. P. E. Influence of bulk graphite density on electrical conductivity. Procedia Engineering 2012, 32, 1100-1106.

(3) Peng, Y.; Chen, J.; Song, A. Y.; Catrysse, P. B.; Hsu, P.-C.; Cai, L.; Liu, B.; Zhu, Y.; Zhou, G.; Wu, D. S. J. N. S. Nanoporous polyethylene microfibres for large-scale radiative cooling fabric. Nature Sustainability 2018, 1 (2), 105. 